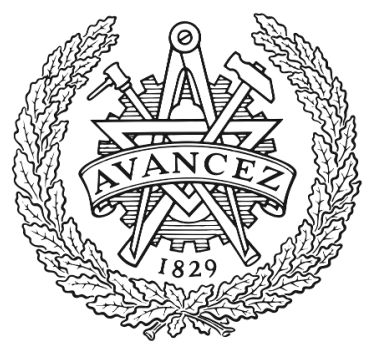

CHALMERS

UNIVERSITY OF TECHNOLOGY

\title{
Lattice-based geometric shaping
}

Downloaded from: https://research.chalmers.se, 2023-04-26 10:07 UTC

Citation for the original published paper (version of record):

Mirani, A., Agrell, E., Karlsson, M. (2020). Lattice-based geometric shaping. European Conference on Optical Communication, ECOC. http://dx.doi.org/10.1109/ECOC48923.2020.9333162

N.B. When citing this work, cite the original published paper. 


\title{
Lattice-based geometric shaping
}

\author{
Ali Mirani( ${ }^{(1)}$, Erik Agrell(2), Magnus Karlsson ${ }^{(1)}$
}

(1) Department of Microtechnology and Nanoscience, Chalmers University of Technology, Gothenburg, Sweden, mirani@chalmers.se

(2) Department of Electrical Engineering, Chalmers University of Technology, Gothenburg, Sweden

\begin{abstract}
Geometrically shaped multidimensional constellations with more than $10^{28}$ points are simulated using fast and low-complexity algorithms without any look-up tables to store the constellation points. At the same symbol error rate, more than $78 \%$ and $114 \%$ reach improvement are demonstrated compared with 4- and 16-QAM, respectively.
\end{abstract}

\section{Introduction}

Constellation shaping has been investigated extensively in recent years to overcome the asymptotic loss $(\approx 1.53 \mathrm{~dB})$ of the conventional quadrature amplitude modulate (QAM) formats in order to increase the transmission rate and distance or reduce the transmitted power ${ }^{[1],[2]}$. Two popular approaches in this area are probabilistic shaping and geometric shaping, both having their own trade-offs and complexities. Probabilistic shaping modifies the probability of transmitted constellation points, and on the other hand, geometric shaping optimizes the constellation points location in Euclidean space. Usually, geometric shaping is considered to be complex to implement because of the large look-up tables required in the transmitter and receiver, and the lack of other demodulation methods than full search ${ }^{[3]}$. However, recent studies have focused on low-complexity implementation of shaping methods with short block length ${ }^{[4]}$.

In this paper, we apply lattice-based constellations with fast modulation and demodulation algorithms whose algorithmic complexity scales with dimensions rather than constellation size. Our multidimensional constellations are simulated in additive white Gaussian noise (AWGN) and nonlinear fiberoptic channels and their performance are compared with the conventional QAM formats at the same spectral efficiencies. As a proof of concept, we are able to modulate and demodulate constellations with a size greater than $10^{28}$ in 24 dimensions.

\section{Theory}

A lattice is an infinite set of points in $N$-dimensional space, which has a regular, periodic structure. It can be constructed using integer linear combinations of basis vectors $\mathbf{g}_{1}, \mathbf{g}_{2}, \cdots, \mathbf{g}_{N} \in \mathbb{R}^{N}$, i.e.,

$$
\Lambda=\left\{\sum_{i=1}^{N} z_{i} \mathbf{g}_{i} \mid z_{i} \in \mathbb{Z}\right\}=\left\{\mathbf{G z} \mid \mathbf{z} \in \mathbb{Z}^{N}\right\} .
$$

The square matrix $\mathbf{G}=\left[\mathbf{g}_{1}, \mathbf{g}_{2}, \cdots, \mathbf{g}_{N}\right]$ is called the generator matrix for the lattice $\Lambda$ and has a non-zero determinant. Based on Eq. (1), all lattices can be constructed as a linear transformation of the integer lattice $\mathbb{Z}^{N}$. This is an important property that can be useful in finding the nearest lattice point to an arbitrary point in $\mathbb{R}^{N}$. In this paper, we focus on the hexagonal $\left(A_{2}\right)$, the checkerboard $\left(D_{4}\right)$, the Gosset $\left(E_{8}\right)$, and the Leech $\left(\Lambda_{24}\right)$ lattices whose description can be found in lattice theory references ${ }^{[5],[6]}$.

In order to use the infinite lattices as a constellation in communication links, they need to be truncated. There are different approaches to truncate a lattice such as sphere cut ${ }^{[7]}$ or cubic cut but the focus of this work is on the Voronoi cut ${ }^{[8]}$ of a lattice. The Voronoi region around a lattice point $\lambda$ is considered as the set of all points in $\mathbb{R}^{N}$ that are closer to $\lambda$ than any other lattice points. Since 0 is always a member of all lattices, its Voronoi region is called the fundamental Voronoi region. By scaling and shifting the fundamental Voronoi region, we can truncate and select a set of lattice points in this region and create the desired constellation. This constellation is called a Voronoi constellation (VC) and can be defined as $\mathcal{C}=\left\{\boldsymbol{\lambda}-\mathbf{a} \mid \boldsymbol{\lambda} \in \Lambda \bigcap\left(\mathbf{a}+V_{r}\right)\right\}$, where $V_{r}$ is the scaled fundamental Voronoi region, $r$ is a scaling factor and $\mathbf{a}$ is a shift vector to avoid any points on the Voronoi cut boundary, which should be selected to minimize the constellation average symbol energy.

In order to use the constellation set $\mathcal{C}$ in a communication system, a fast and low complexity algorithm is needed to modulate and demodulate the transmitted and received data. Such algorithms have been proposed for VCs by Conway and Sloane $e^{[9],[10]}$ and in this paper we call them lattice-based modulation and demodulation algorithms ${ }^{[11]}$. The core part of these algorithms is known as the closest point algorithm. The demodulation algorithm is faster and less complex than performing maximum likelihood (ML) estimation for our geometrically shaped constellations because i) it does not require a comparison with all constellation points, ii) it does not need a look-up table to store the constellation points and iii) its algorithmic 


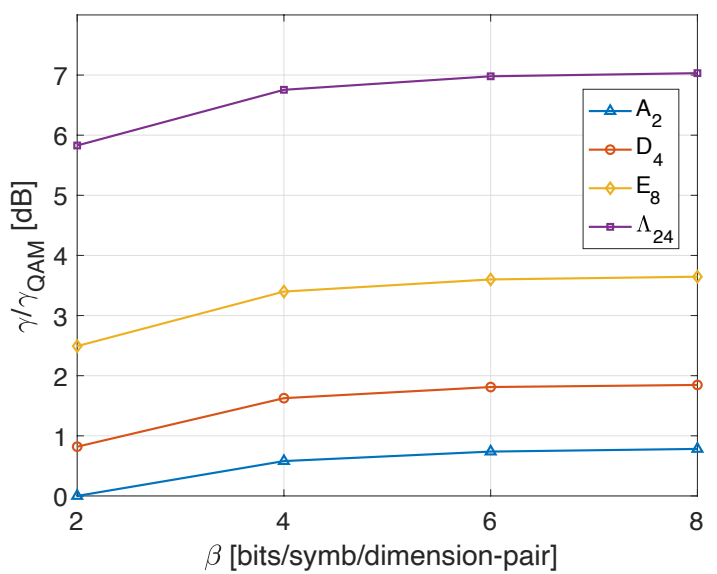

Fig. 1: Comparison of asymptotic power efficiency of multidimensional VCs with respect to QAM formats at different spectral efficiencies

complexity scales with dimensionality rather than the constellation size. Furthermore, it has been shown that the demodulation algorithm performs close to optimum as the constellation size or the signal to noise ratio (SNR) increases ${ }^{[11]}$.

\section{Figures of merit}

The asymptotic power efficiency (APE) of a constellation is defined as $\gamma=d_{\min }^{2} /\left(4 E_{b}\right)$ where $d_{\min }$ is the minimum Euclidean distance between the constellation points and $E_{b}$ is the average constellation energy per bit. This parameter depends on the constellation geometry and at the same bit rate indicates the asymptotic power gain of the constellation to reach a certain symbol error rate (SER).

The spectral efficiency (SE) indicates the number of transmitted bits per symbol per dimension-pair, and it is defined as $\beta=\log _{2}(M) /(N / 2)$, where $M$ is the constellation size. In fiber-optic communication, one polarization of light is considered to have a pair of dimensions.

In fig. 1, the APE of VCs in different dimensions with respect to QAM formats $\left(\gamma_{Q A M}=3 \log _{2} M /(2(M-1))\right)^{[12]}$ at various SEs is

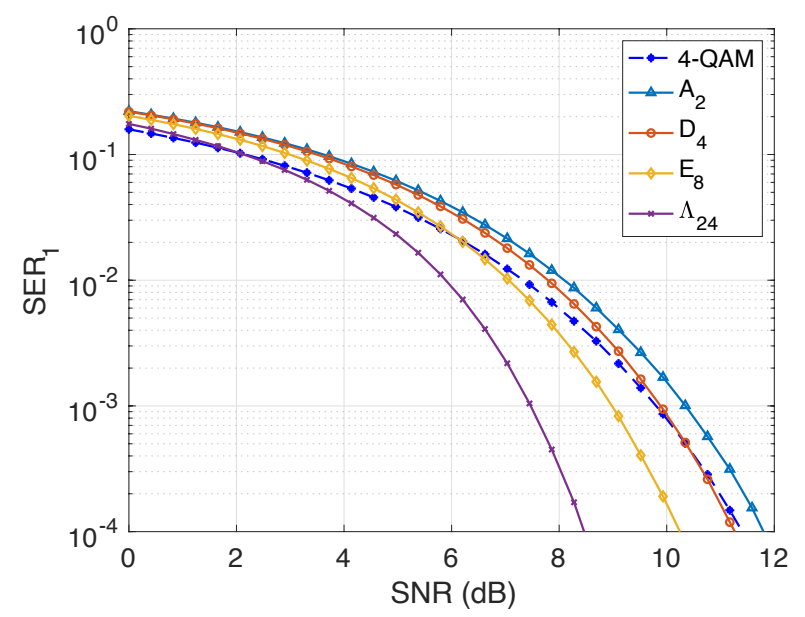

(a) $\beta=2$ bits/symb/dimension-pair
Tab. 1: Shaping gain and coding gain for $\mathrm{VCs} s^{[8],[13]}$

\begin{tabular}{|c|c|c|c|c|c|}
\hline Lattice & $\mathbb{Z}^{N}$ & $A_{2}$ & $D_{4}$ & $E_{8}$ & $\Lambda_{24}$ \\
\hline$\gamma_{c}(\mathrm{~dB})$ & 0 & 0.62 & 1.51 & 3.01 & 6.02 \\
\hline$\gamma_{s}(\mathrm{~dB})$ & 0 & 0.17 & 0.37 & 0.65 & 1.03 \\
\hline
\end{tabular}

plotted. As shown in this figure, by increasing $\beta$, the curves saturate to a value which corresponds to the product of the shaping gain $\left(\gamma_{s}\right)$ of the constellation boundary (Voronoi cut) and the coding gain $\left(\gamma_{c}\right)$ of the lattice. These values are shown in Tab. 1.

To have a fair comparison between the SER performance of multidimensional modulation formats, their SER should be normalized per dimension ${ }^{[14]}$. This can be done by transforming the multidimensional SER $\left(S E R_{N}\right)$ to the one-dimensional SER $\left(S E R_{1}\right)$ by

$$
S E R_{1}=1-\sqrt[N]{1-S E R_{N}}
$$

\section{AWGN channel}

The setup includes generating multidimensional symbols $(\mathrm{x})$ using a lattice-based modulation algorithm ${ }^{[11]}$ and adding multidimensional AWGN to the transmitted symbol by the channel. Thus, the received point is $\mathbf{y}=\mathbf{x}+\mathbf{n}$, where $\mathbf{n}$ is the AWGN with covariance matrix of $\Sigma=\sigma_{0}^{2} \mathbb{I}_{N \times N}$ and $\sigma_{0}^{2}=N_{0} / 2$ is the noise variance per dimension and $\mathbb{I}_{N \times N}$ is the identity matrix. Also, the SNR is defined as $S N R=E_{s} /\left(N \cdot N_{0} / 2\right)$, where $E_{s}$ is the average symbol energy. In the receiver, we use the latticebased demodulation algorithm ${ }^{[11]}$ to find the closest constellation point. Finally, Monte Carlo simulations are used to estimate the normalized SER performance of VCs. The results are compared with QAM constellations with the optimum $\mathrm{ML}$ receiver. In fig. 2, SEs of 2 and 8 bits/symb/dimension-pair are simulated and, in the AWGN channel, gains of up to $4 \mathrm{~dB}$ can be achieved by increasing the SE and dimensions at $S E R_{1}=10^{-4}$. As can be seen in fig. 2, for relatively low SNR values, VCs perform worse than QAM because they have a higher number of neigh-

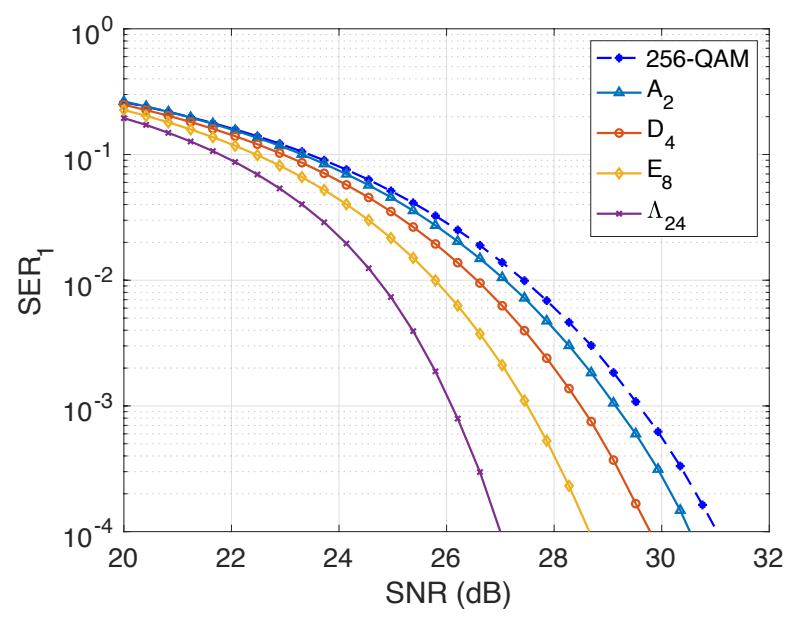

(b) $\beta=8$ bits/symb/dimension-pair

Fig. 2: Normalized SER comparison of VCs in different dimensions by QAM formats with an optimum ML receiver. 


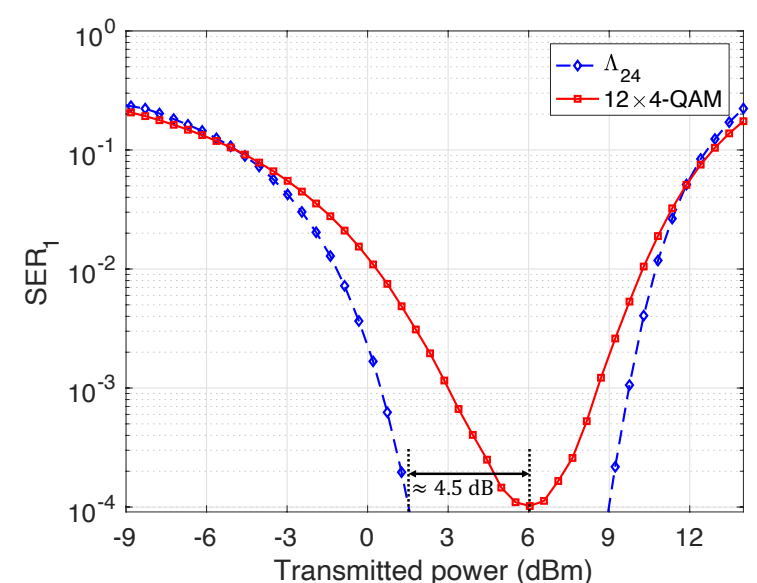

Fig. 3: Normalized SER comparison after 72 spans of fiber at $\beta=2$ bits/symb/pol, and 6 WDM channels.

bors at distance $d_{\text {min }}$.

In fig. $2 \mathrm{~b}$ with the $\Lambda_{24}$ lattice, using the latticebased modulation and demodulation algorithms, we are able to transmit and receive symbols from a constellation with a size of $M=2^{96} \approx 7.92 \times 10^{28}$. This demonstrates the strength of VCs, as any unstructured geometric shaping scheme of the same size would be infeasible due to the memory requirement for storing the constellation and the complexity of $\mathrm{ML}$ decoding.

\section{Nonlinear fiber channel}

We investigate the performance of multidimensional VCs in a nonlinear dispersive fiber-optic channel. We modulate the polarization, wavelength, and in-phase and quadrature components of the optical field. Our simulation setup consists of a transmitter generating 28 GBaud channels in each wavelength and polarization in a wavelength-division multiplexing (WDM) system with $50 \mathrm{GHz}$ spacing. The multidimensional VC symbols are mapped to multiple 4-dimensional symbols in each wavelength. The optical channel includes a fiber link with multiple $80 \mathrm{~km}$ spans having parameters of attenuation $0.2 \mathrm{~dB} / \mathrm{km}$, dispersion $17 \mathrm{ps} / \mathrm{nm} / \mathrm{km}$, and nonlinearity $1.3 \mathrm{~W}^{-1} \mathrm{~km}^{-1}$. The

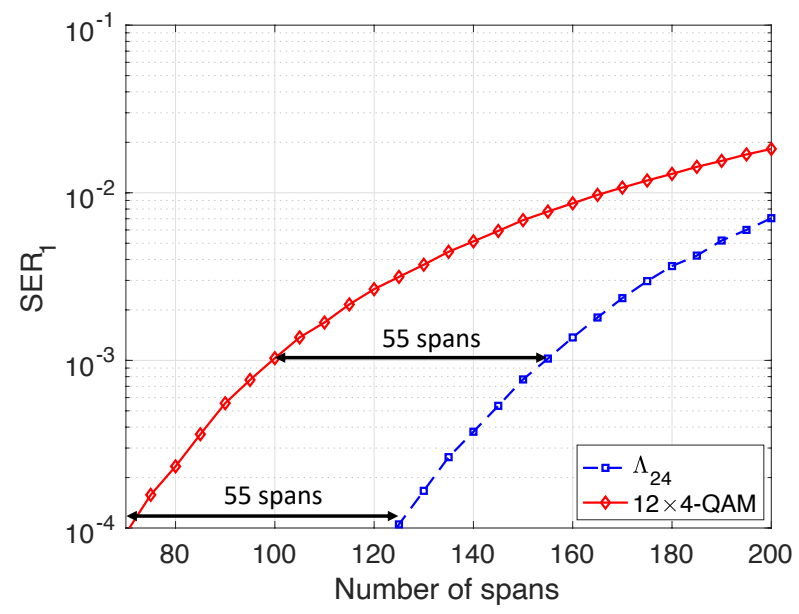

(a) $\beta=2$ bits $/ \mathrm{symb} / \mathrm{pol}, 6$ WDM channels split-step Fourier method is used to simulate the propagation of light inside the fiber link. At the end of each span, an optical amplifier with a noise figure of $5 \mathrm{~dB}$ compensates the fiber losses. On the receiver side, after dispersion compensation for the whole bandwidth and total fiber length, polarization demultiplexing and phase estimation are performed by $1.5 \%$ overhead 4-QAM pilot symbols. Then, 4-dimensional symbols are combined to form a multidimensional received point and the lattice-based demodulation algorithm ${ }^{[11]}$ is applied to estimate the transmitted constellation point.

In fig. 3, we compare the normalized SER between a $\Lambda_{24}$-based VC and 4-QAM with ML detection over a nonlinear dispersive fiber link at the same SEs. As shown in fig. 3, power gains of $4.5 \mathrm{~dB}$ are achieved at a $S E R_{1}$ of $10^{-4}$. In fig. 4, the SERs are calculated close to the optimum transmitted power for each transmission distance. At a $S E R_{1}$ of $10^{-4}$, the transmission distance can be increased by more than 55 and 16 spans at SEs of 2 and 4 bits/symb/pol, respectively.

\section{Conclusions}

In this work, lattice-based Voronoi constellations have been suggested as a multidimensional geometric shaping approach in optical communication systems. Fast and low-complexity modulation and demodulation schemes without storing the constellation points in a look-up table provide a significant advantage over other constellation shaping methods which might have long block lengths. More than $78 \%$ and $114 \%$ of transmission reach improvement compared to 4-QAM and 16-QAM formats are shown over the nonlinear fiber-optic channel, respectively.

\section{Acknowledgements}

We wish to acknowledge financial support from the Knut and Alice Wallenberg foundation as well as the Swedish Research Council (VR) in projects 2017-03702 and 2019-04078.

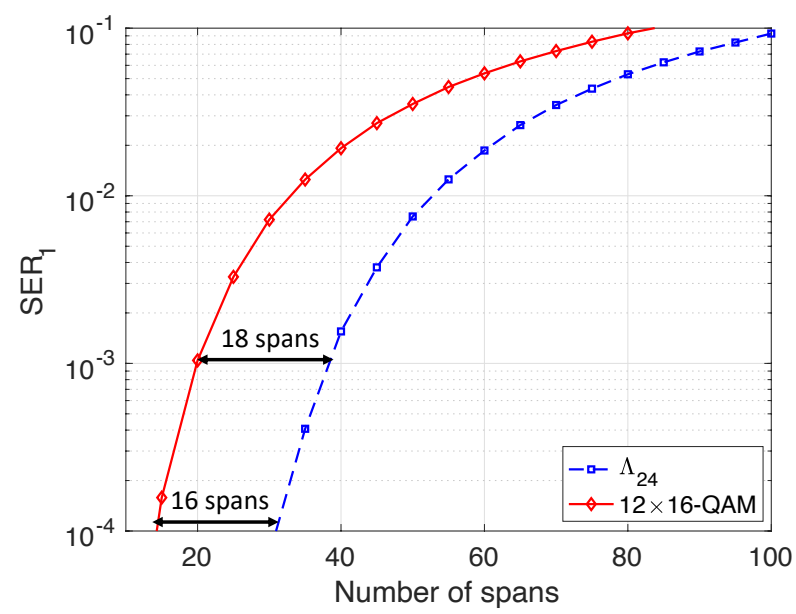

(b) $\beta=4$ bits/symb/pol, 6 WDM channels

Fig. 4: Normalized SER comparison of the multidimensional VCs with QAM formats at their optimum transmission power. 


\section{References}

[1] Z. Qu and I. B. Djordjevic, "On the probabilistic shaping and geometric shaping in optical communication systems", IEEE Access, vol. 7, pp. 21 454-21 464, 2019.

[2] S. Zhang and F. Yaman, "Constellation design with geometric and probabilistic shaping", Optics Communications, vol. 409, pp. 7-12, 2018.

[3] J. Cho and P. J. Winzer, "Probabilistic constellation shaping for optical fiber communications", Journal of Lightwave Technology, vol. 37, no. 6, pp. 1590-1607, 2019.

[4] T. Matsumine, T. Koike-Akino, D. S. Millar, K. Kojima, and K. Parsons, "Short lattice-based shaping approach exploiting non-binary coded modulation", ECOC, 2019.

[5] S. I. Costa, F. Oggier, A. Campello, J.-C. Belfiore, and E. Viterbo, Lattices Applied to Coding for Reliable and Secure Communications. Springer, 2017.

[6] J. H. Conway and N. J. A. Sloane, Sphere packings, lattices and groups. Springer Science \& Business Media, 2013, vol. 290.

[7] D. S. Millar, T. Koike-Akino, S. Ö. Arık, K. Kojima, K. Parsons, T. Yoshida, and T. Sugihara, "High-dimensional modulation for coherent optical communications systems", Optics express, vol. 22, no. 7, pp. 8798-8812, 2014.

[8] G. D. Forney, "Multidimensional constellations. II. voronoi constellations", IEEE Journal on Selected Areas in Communications, vol. 7, no. 6, pp. 941-958, 1989.

[9] J. Conway and N. Sloane, "Fast quantizing and decoding and algorithms for lattice quantizers and codes", IEEE Transactions on Information Theory, vol. 28, no. 2, pp. 227232, 1982.

[10] — "A fast encoding method for lattice codes and quantizers", IEEE Transactions on Information Theory, vol. 29, no. 6, pp. 820-824, 1983.

[11] A. Mirani, E. Agrell, and M. Karlsson, Low-complexity geometric shaping, 2020. arXiv: 2008.10330 [eess.SP].

[12] E. Agrell and M. Karlsson, "Power-efficient modulation formats in coherent transmission systems", Journal of Lightwave Technology, vol. 27, no. 22, pp. 5115-5126, 2009.

[13] S. Benedetto and E. Biglieri, Principles of digital transmission: with wireless applications. Springer Science \& Business Media, 1999.

[14] R. Zamir, Lattice Coding for Signals and Networks: A Structured Coding Approach to Quantization, Modulation, and Multiuser Information Theory. Cambridge University Press, 2014. 